OPEN ACCESS

Edited by: Ilyas Sahin, Brown University, United States

Reviewed by: Muhammet Sayan, Gazi University, Turkey

Bo Zhang,

Shanghai Jiaotong University, China

*Correspondence: Yanru Qin

yanruqin@163.com

${ }^{\dagger}$ These authors share first authorship

Specialty section: This article was submitted to Cancer Molecular Targets and Therapeutics,

a section of the journal

Frontiers in Oncology

Received: 08 December 2020 Accepted: 22 April 2021 Published: 11 May 2021

Citation:

Li J, Liang $H, H e J$, Sui $X$ and Qin Y (2021) Anlotinib Combined With Chemotherapy for Recurrence of Pulmonary Sarcomatoid Cancer Previously Surgically Treated: A Case Report and Literature Review.

Front. Oncol. 11:639168. doi: 10.3389/fonc.2021.639168

\section{Anlotinib Combined With Chemotherapy for Recurrence of Pulmonary Sarcomatoid Cancer Previously Surgically Treated: A Case Report and Literature Review}

\author{
Jing $\mathrm{Li}^{\dagger}$, Hejun Liang ${ }^{\dagger}$, Jian He, Xin Sui and Yanru Qin * \\ Department of Oncology, The First Affiliated Hospital of Zhengzhou University, Zhengzhou, China
}

Background: Pulmonary sarcomatoid cancer (PSC) is a very rare subtype of poorly differentiated non-small-lung-cancer (NSCLC) with very poor prognosis. To date, the optimal treatment for PSC has not been elucidated, and the efficacy of anlotinib in PSC has not been previously reported.

Case Presentation: A 77-year-old male patient was admitted with cough, expectoration, and blood-stained sputum for one month. CT showed a soft mass in the inferior lobe of the right lung, which was diagnosed as spindle cell carcinoma (PSC) by histopathology. A videothoracoscopic right lower lobectomy and mediastinal lymph node dissection procedure was performed on the patient, but the disease recurred one month after surgery. The patient was then given first-line chemotherapy with gemcitabine and albumin paclitaxel for one cycle, but the disease continued to progress. The patient then received anlotinib combined with second-line chemotherapy (dacarbazine and cis-platinum) for six cycles, and the response reached complete remission (CR). Then the patient was given maintenance therapy with anlotinib alone, and the disease was still stable at the most recent reexamination. Progression-free survival (PFS) has lasted for more than two years, without any intolerable toxicity.

Conclusion: This postoperative recurrent PSC patient achieved significant clinical benefits with anlotinib treatment. Our findings provide direct evidence of the efficacy of anlotinib in PSC. More studies are needed to confirm our observation.

Keywords: pulmonary sarcomatoid cancer (PSC), anlotinib, chemotherapy, targeted therapy, case report

\section{INTRODUCTION}

Sarcomatoid carcinoma (SC) is a rare malignancy with a combination of epithelial and sarcoma or sarcoma-like components $(1,2)$. Pulmonary sarcomatoid carcinomas (PSCs) are a heterogeneous group of non-small-cell lung carcinomas containing five subgroups, namely pleomorphic carcinoma, spindle cell carcinoma, giant cell carcinoma, carcinosarcoma, and pulmonary blastoma, which comprise $0.1 \%$ to $0.4 \%$ of all pulmonary malignancies (3). This malignancy has been reported to 
have very poor prognosis and shorter overall survival than other NSCLCs (4). Epidemiologically, the average age of diagnosis is 60 years. Because SC is most commonly observed in heavy smokers, there is also a male predominance (5). PSCs have been shown to respond poorly to platinum-based chemotherapy, and thus there is an urgent need for new and effective therapeutic options (6).

Anlotinib hydrochloride is a novel multitarget tyrosine kinase inhibitor (TKI) that has a significant inhibitory effect on tumor angiogenesis and proliferative signaling by selectively targeting VEGFR-2,-3 and FGFR-1,-2,-3,-4 and suppressing the activity of PDGFR $\alpha / \beta$, C-has Kit, Ret, Aurora-B, C-FMS, and discoidin domain receptor (DDR1) (7). In preclinical studies, anlotinib has shown significant antitumor activity against a variety of xenograft models (7). In a phase III study called ALTER 0303, anlotinib showed a significant increase in OS and PFS in patients with advanced NSCLC as a third or later line of treatment (8). In a phase II study for patients with refractory soft-tissue sarcoma (STS), anlotinib also showed remarkable antitumor activity; overall, the 12 -week progression-free rate $\left(\mathrm{PFR}_{12}\right.$ weeks $)$ and the overall response rate (ORR) were $68 \%$ and $13 \%$, respectively, and the PFS and overall survival (OS) were 5.6 months and 12 months, respectively (9).

Here, we present a case report that showed an excellent therapeutic effect of anlotinib-combined chemotherapy against this malignancy.

\section{CASE PRESENTATION}

A 77-year-old male was admitted to our hospital complaining of coughing, expectoration, and blood-stained sputum for one month on September 11, 2018. There were no symptoms of fever, shortness of breath, chest stuffiness, or chest pain. He had a history of smoking for 40 years and smoked up to 20-25 cigarettes each day. In addition, he also had a history of stage 3 hypertension for 20 years and nifedipine delayed-release tablets were used to control blood pressure. Blood chemistry and tumor markers were within normal limits. A contrast-enhanced CT scan of the chest and abdomen showed a soft mass $(42 \times 38 \mathrm{~mm})$ in the inferior lobe of the right lung without any lymph nodes or distant metastasis (Figure 1). A videothoracoscopic right lower lobectomy and mediastinal lymph node dissection procedure were performed on the patient on August 3, 2018. The postoperative pathological examination revealed a pulmonary sarcomatoid carcinoma with a size of $3.2 \times 2.5 \times 1.5 \mathrm{~cm}$ and with negative surgical margins of the bronchus and vessels. The subtype of PSC was confirmed as spindle cell carcinoma by the pathologist. A total of 37 lymph nodes were retrieved, including the second, fourth, seventh, tenth, and eleventh groups of lymph nodes, and all of them were negative. The TNM staging was T2aN0M0 IB according to the $8^{\text {th }}$ edition of the AJCC/UICC TNM staging system for lung cancer. Immunohistochemistry showed that the cells were stained positive for EMA(focal + ), CK8/18(focal+), TTF-1(focal+), Ki-67(70\%), CD34(focal+), $\operatorname{CD} 31(+)$, and $\operatorname{SMA}(+)$, and negative for $\operatorname{AE} 1 / \operatorname{AE} 3(-)$, NapsinA(-), CK5/6(-), P40(-), P63(-), S-100(-), ERG(-), and Desmin(-) (Figure 2). Next-generation sequencing (NGS) showed 10 somatic mutations in BRAD1 [p.E355K (c.G1083A)], EPCAM [p.F20S(c.T59C)], EPHA3 [p.R274Q (c.G821A)], FGFR2 [p.A389V(c.C1166T)], KRAS [p.G12C (c.G34T)], RAC1 [p.A59G(c.C176G)], RAD54L [p.R688C (c.C2062T)], SMRCA4 [p.A733V(c.C2198T)], TERT [c.C124T], and TP53 genes [p.R80K(c.G839A)]. Nevertheless, common mutations such as in EGFR, ALK, and ROS-1 genes were not found by NGS. The tumor mutational burden (TMB) was 9.7 mutations/Mb and the microsatellite status was microsatellite stability (MSS). Unfortunately, follow-up CT reexamination one month after surgery demonstrated disease recurrence in the mediastinum and right pleural effusion (Figure 3). Then the patient underwent one course of first-line chemotherapy consisting of gemcitabine and albumin paclitaxel. However, the coughing and expectoration persisted, and there was no significant relief after discharge. Because new symptoms of chest stuffiness and fatigue increased gradually, the patient was readmitted for a CT scan, which showed that the tumor had progressed in the mediastinum, the right pleura, and bronchial stump, and there was a new distant metastasis in the upper abdomen (Figures 4-A1, B1, C1, D1). The response evaluation was progressive disease (PD). Therefore, we changed the regimen to anlotinib combined with second-line

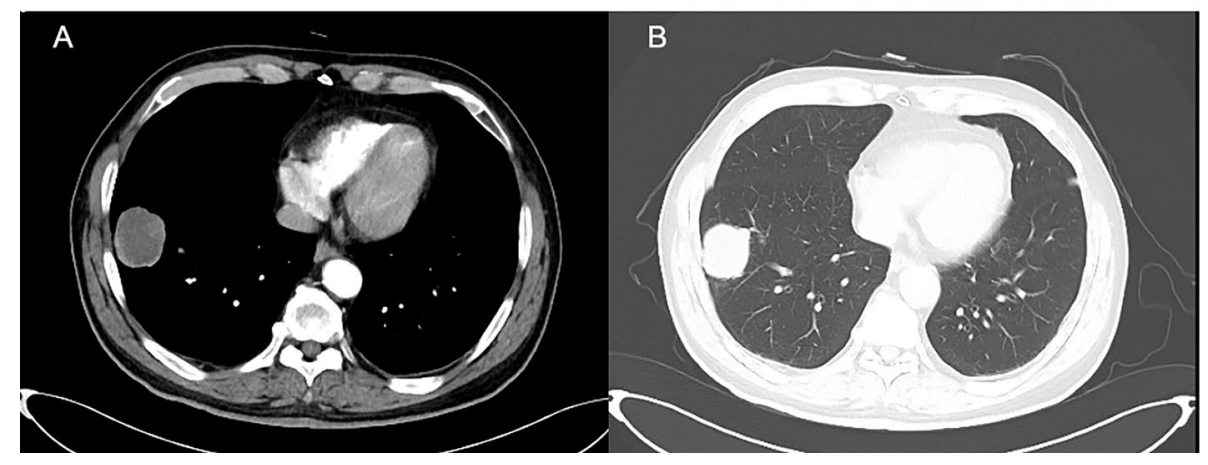

FIGURE 1 | Occupation in the right inferior lobe of lung. (A) lung window, (B) mediastinal window. 


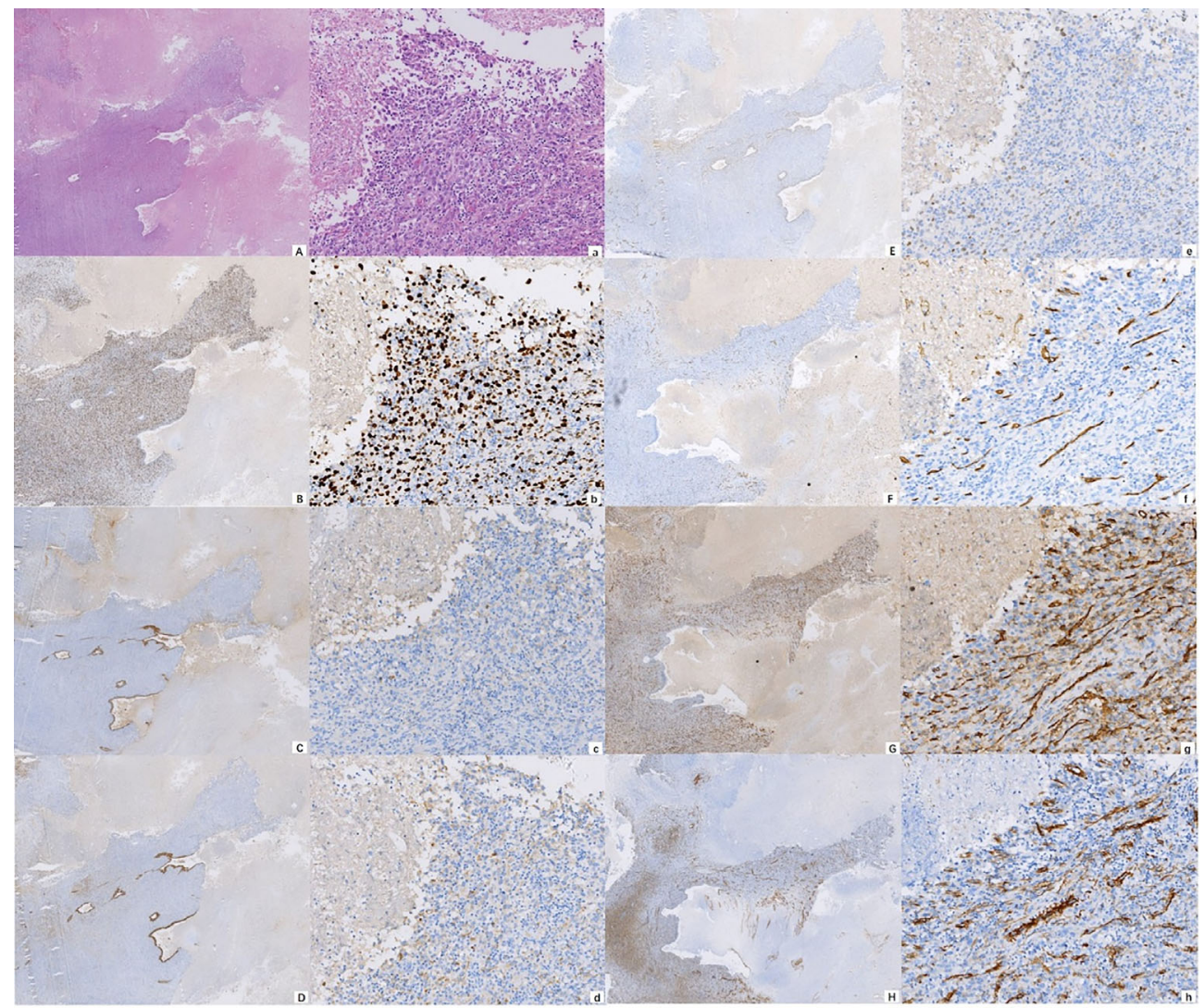

FIGURE 2 | Photomicrograph showing the cytomorphological finds (A) H\&E $\times 10$; a H\&E $\times 100$ ) and immunohistochemical staining of the malignancy, positive for Ki-

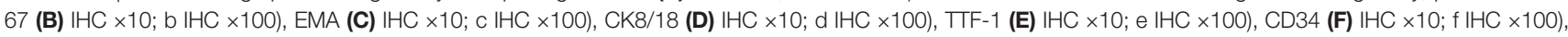
CD31 (G) $\Vdash H C \times 10 ; g I H C \times 100)$, and SMA (H) $\Vdash H C \times 10 ; ~ h ~ I H C \times 100)$.

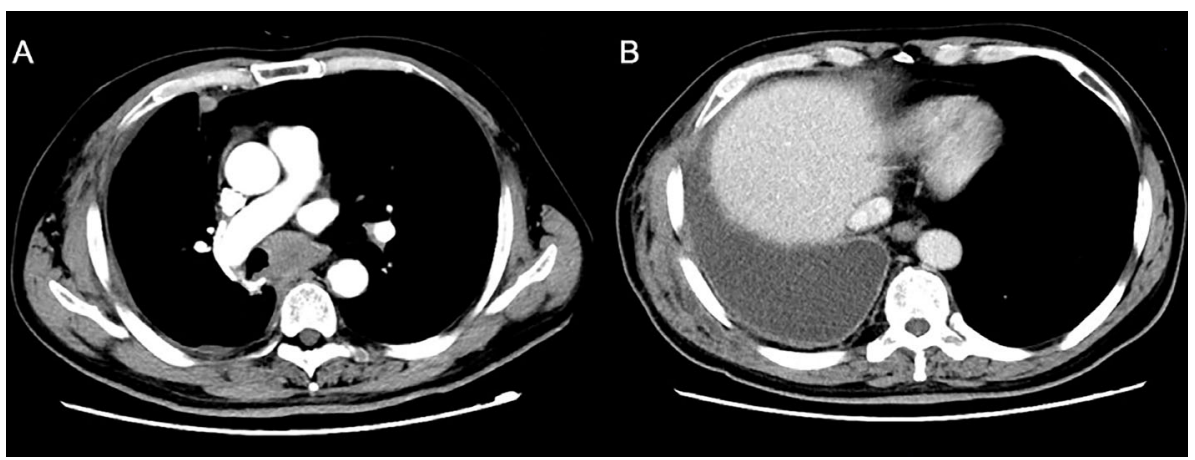

FIGURE 3 | A reexamination CT scan one month after surgery showed new soft tissue nodules in the mediastinum.

chemotherapy consisting of dacarbazine and cis-platinum. After one cycle of treatment, symptoms of coughing, expectoration, chest stuffiness, and fatigue were significantly relieved. After two cycles of treatment, the symptoms disappeared substantially. Follow-up CT reexamination after two cycles of treatment showed that the tumor regressed significantly and the response evaluation was partial remission (PR) (Figures 4-A2, B2, C2, D2). After four cycles of treatment, a CT scan demonstrated that the tumor remained in remission, and the response evaluation remained PR (Figure 4-A3, B3, C3, D3). PET-CT was performed for therapeutic response assessment, and the results showed CR after the completion of six cycles of treatment (Figure 5). 


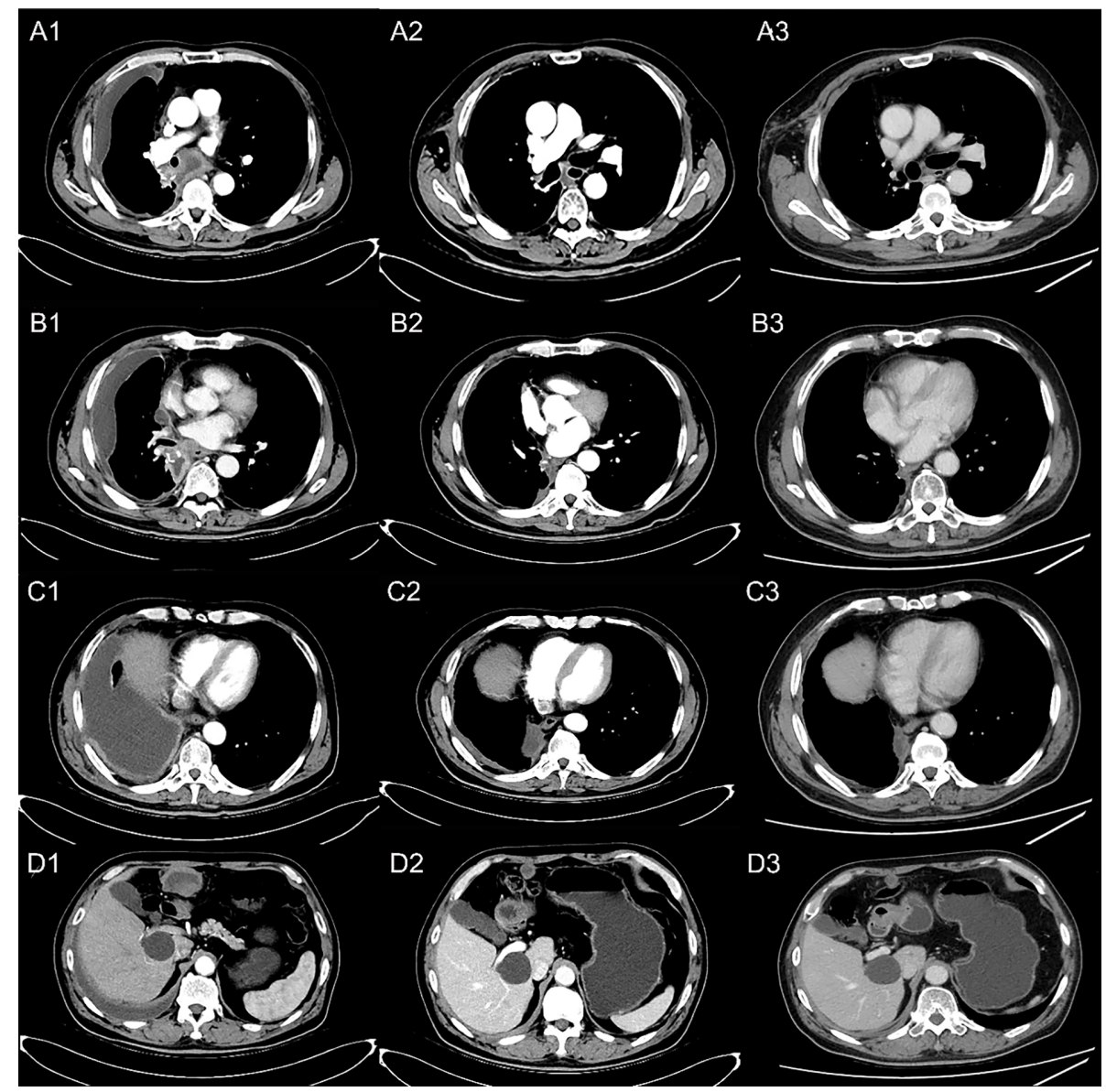

FIGURE 4 | Reexamination results showing tumor regression by contrast-enhanced CT scan. (A) Occupation in mediastinum; (B) thickening of the right pleura; (C) soft tissue by the side of the right bronchial stump; (D) metastatic lesion on the right upper abdomen. (A1, B1, C1, D1) PD after one cycle of first-line chemotherapy; (A2, B2, C2, D2) disease PR after two cycles of the second-line treatment; (A3, B3, C3, D3) disease remaining as PR after four cycles of the second-line treatment.

Subsequently, the patient continued taking anlotinib as a maintenance treatment. In the most recent reexamination (more than two years after therapy), the disease was still stable, and there was no intolerable toxicity.

\section{DISCUSSION}

PSC is a rare malignancy of NSCLC and has a poorly differentiated and aggressive signature (10). At present, there are no standard treatments for the malignancy. Platinum-based chemotherapy for NSCLC has long been used for PSC patients, but prognosis has remained poor and the median OS is no more than nine months for metastatic disease according to previous studies (4). In previous clinical practice, we treated some PSC patients using platinum-based chemotherapies such as pemetrexed combined with cisplatin or gemcitabine combined with cisplatin, but the responses were disappointing. We also used gemcitabine and taxanes, which are therapeutic regimens for STS. Unfortunately, the response has still been unsatisfactory. Therefore, it is necessary to identify new active agents to improve the therapeutic effect for PSC patients. Sarcomatoid carcinoma is a malignancy with a combination of epithelial and sarcoma or sarcoma-like components. We therefore used a new chemotherapy agent comprising dacarbazine and cis-platinum. Dacarbazine is a standard anti-sarcoma agent with classically known activity, generally reserved as a later line of therapy, and cis-platinum is a major component of NSCLC treatment $(11,12)$. Eventually, the disease achieved CR after six cycles of the second-line treatment.

The programmed death-1 (PD-1) and programmed death ligand-1 (PD-L1) axis is a classical immune checkpoint pathway. Tumor cells utilize this immunosuppressive co-signal to evade immune defenses in the tumor microenvironment. A blockade of the PD-1/PD-L1 axis has restored effector T-cell function and enhanced antitumor immune activity in preclinical studies (13). Consistently, clinical trials of PD-1/PD-L1 signal-blockade agents have shown prominent antitumor efficacy in several malignancies including NSCLC, and the outcome is positively related to the expression level of PD-1 (14-16). With regard to PSC, previous studies have shown that the expression level of PD-L1 is high (17). 
A
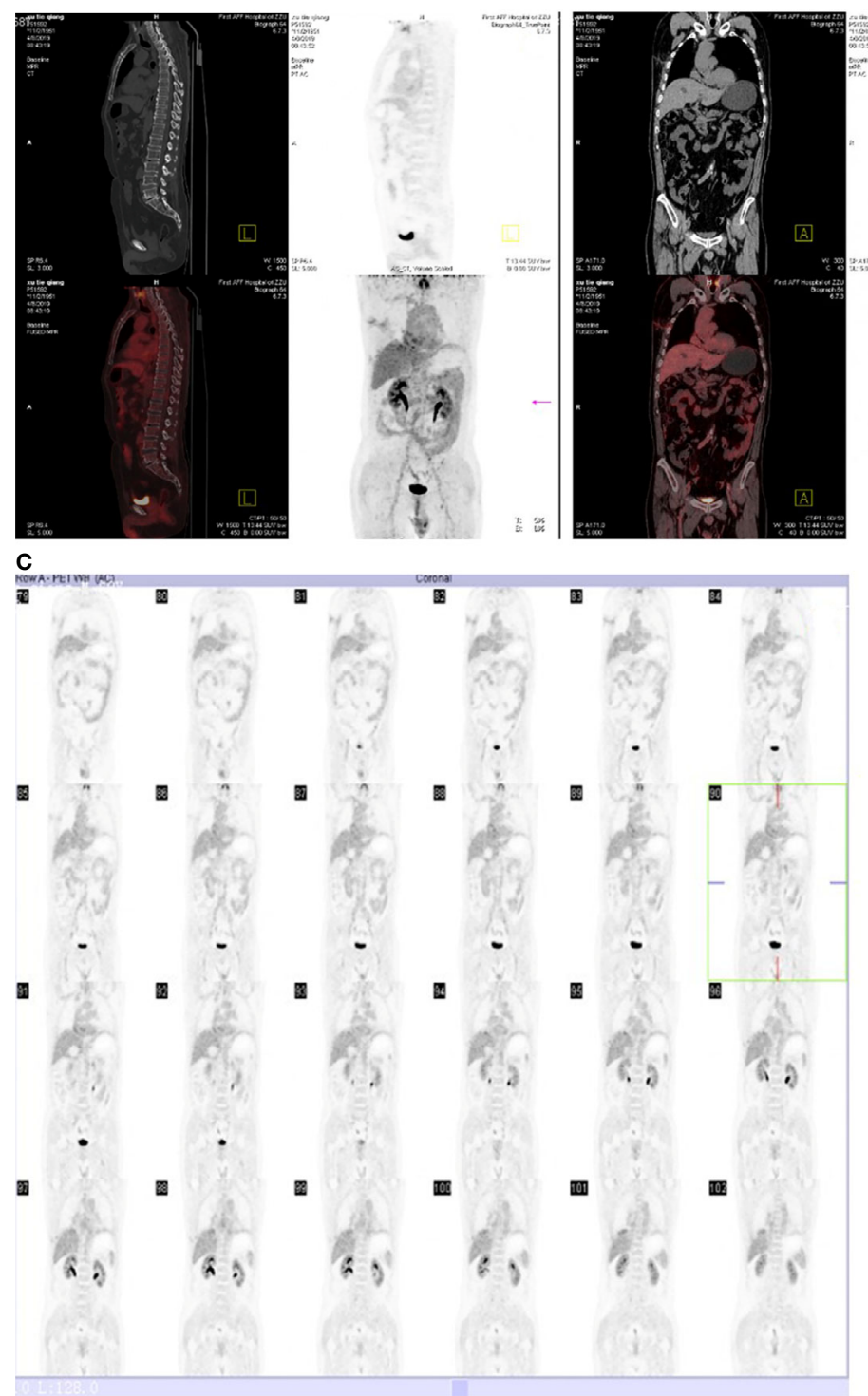

B
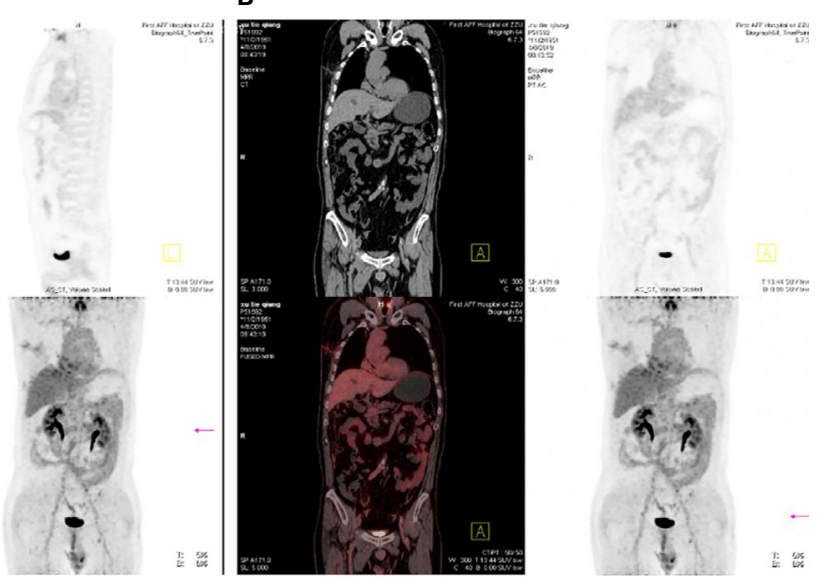

FIGURE 5 | Reexamination PET-CT scan showing CR after six cycles of second-line treatment.

Therefore, we tested PD-L1 expression in this patient. However, as the test results were negative, a PD-1 inhibitor was not used.

Recently, several studies have focused on the genetic features of this disease and revealed a wide range of alterations, including TP53, EGFR, KRAS, PIK3CA, STK11, BRCA1/BRCA2, and IDH1 mutations; MET exon 14 mutations; and MET, ALK, EGFR, CDK4, and MDM2 amplifications, some of which are associated with targeted therapy through the MET receptor pathway or MAP-kinase pathway $(3,6)$. In this case, ten somatic mutations including two common mutations, specifically TP53 and KRAS, along with other occasional mutations, specifically BRAD1, EPCAM, EPHA3, FGFR2, RAC1, RAD54L, SMARCA4, and TERT, were found by NGS. Unfortunately, common mutations of EGFR, ALK, and ROS-1 genes were not found.
Anlotinib is a multitargeting TKI that can significantly inhibit tumor proliferation, vasculature, and the tumor micro environment by selectively targeting VEGFR-2,-3, FGFR-1,-2,-3,4, PDGFR $\alpha / \beta$, C-Kit, Ret, Aurora-B, c-FMS, and DDR1 (7). A phase I study showed that anlotinib has promising anti-tumor potential against a variety of tumors, including STS and NSCLC (18). At present, anlotinib has been approved as a second- or laterline treatment for STS and third- or later-line treatment for NSCLC based on the ALTER 0203 and ALTER 0303 clinical trials in China $(8,9)$. Considering that NGS for this patient revealed a few gene mutations, including of the FGFR gene, we combined chemotherapy with anlotinib, which selectively targets FGFR, and achieved prominent tumor regression and a long PFS without evident toxicity. 


\section{CONCLUSION}

In summary, we combined chemotherapy with a small molecular TKI known as anlotinib for a PSC patient and achieved a long PFS and remarkable tumor regression. To our knowledge, this is the first case report of the successful treatment of this disease by using this combined treatment. Future clinical trials are expected to validate these new treatments for PSC patients.

\section{DATA AVAILABILITY STATEMENT}

All datasets generated for this study are included in the article.

\section{ETHICS STATEMENT}

Studies involving human participants were reviewed and approved by the Ethics Committee of the First Affiliated Hospital of Zhengzhou University of Henan Province. The patients/participants provided their written informed

\section{REFERENCES}

1. Huang SY, Shen SJ, Li XY. Pulmonary Sarcomatoid Carcinoma: A Clinicopathologic Study and Prognostic Analysis of 51 Cases. World J Surg Oncol (2013) 11:252. doi: 10.1186/1477-7819-11-252

2. Sayan M, Bas A, Valiyev E, Celik A, Kurul IC, Aribas OK, et al. Prognostic Factors for Sarcomatoid Carcinomas of Lung: A Single-Centre Experience. Lung India (2020) 37(6):506-10. doi: 10.4103/lungindia.lungindia_525_19

3. Liu X, Jia Y, Stoopler MB, Shen Y, Cheng H, Chen J, et al. Next-Generation Sequencing of Pulmonary Sarcomatoid Carcinoma Reveals High Frequency of Actionable MET Gene Mutations. J Clin Oncol (2016) 34(8):794-802. doi: 10.1200/JCO.2015.62.0674

4. Vieira T, Girard N, Ung M, Monnet I, Cazes A, Bonnette P, et al. Efficacy of FirstLine Chemotherapy in Patients With Advanced Lung Sarcomatoid Carcinoma. J Thorac Oncol (2013) 8(12):1574-7. doi: 10.1097/01.JTO.0000437008.00554.90

5. Blaukovitsch M, Halbwedl I, Kothmaier H, Gogg-Kammerer M, Popper HH. Sarcomatoid Carcinomas of the Lung-are These Histogenetically Heterogeneous Tumors? Virchows Arch (2006) 449(4):455-61. doi: 10.1007/s00428-006-0256-8

6. Pecuchet N, Vieira T, Rabbe N, Antoine M, Blons H, Cadranel J, et al. Molecular Classification of Pulmonary Sarcomatoid Carcinomas Suggests New Therapeutic Opportunities. Ann Oncol (2017) 28(7):1597-604. doi: 10.1093/annonc/mdx162

7. Lin B, Song X, Yang D, Bai D, Yao Y, Lu N. Anlotinib Inhibits Angiogenesis Via Suppressing the Activation of VEGFR2, PDGFRbeta and FGFR1. Gene (2018) 654:77-86. doi: 10.1016/j.gene.2018.02.026

8. Han B, Li K, Wang Q, Zhang L, Shi J, Wang Z, et al. Effect of Anlotinib as a Third-Line or Further Treatment on Overall Survival of Patients With Advanced non-Small Cell Lung Cancer: The ALTER 0303 Phase 3 Randomized Clinical Trial. JAMA Oncol (2018) 4(11):1569-75. doi: 10.1001/jamaoncol.2018.3039

9. Chi Y, Fang Z, Hong X, Yao Y, Sun P, Wang G, et al. Safety and Efficacy of Anlotinib, a Multikinase Angiogenesis Inhibitor, in Patients With Refractory Metastatic Soft-Tissue Sarcoma. Clin Cancer Res (2018) 24(21):5233-8. doi: 10.1158/1078-0432.CCR-17-3766

10. Terra SB, Jang JS, Bi L, Kipp BR, Jen J, Yi ES, et al. Molecular Characterization of Pulmonary Sarcomatoid Carcinoma: Analysis of 33 Cases. Mod Pathol (2016) 29(8):824-31. doi: 10.1038/modpathol.2016.89

11. Garcia-Del-Muro X, Lopez-Pousa A, Maurel J, Martin J, Martinez-Trufero J, Casado A, et al. Randomized Phase II Study Comparing Gemcitabine Plus Dacarbazine Versus Dacarbazine Alone in Patients With Previously Treated consent to participate in this study. Written informed consent was obtained from the individual(s) for the publication of any potentially identifiable images or data included in this article.

\section{AUTHOR CONTRIBUTIONS}

JL: treatment of the patient, clinical data collection, and manuscript preparation. HL: literature research and manuscript preparation. JH: treatment of the patient and clinical data collection. XS: treatment of the patient and clinical data collection. YQ: treatment of the patient and guarantee of the integrity of the whole research process. All authors contributed to the article and approved the submitted version.

\section{ACKNOWLEDGMENTS}

We thank LetPub (www.letpub.com) for its linguistic assistance during the preparation of this manuscript.

Soft Tissue Sarcoma: A Spanish Group for Research on Sarcomas Study. J Clin Oncol (2011) 29(18):2528-33. doi: 10.1200/JCO.2010.33.6107

12. D'Adamo DR, Dickson MA, Keohan ML, Carvajal RD, Hensley ML, Hirst CM, et al. A Phase II Trial of Sorafenib and Dacarbazine for Leiomyosarcoma, Synovial Sarcoma, and Malignant Peripheral Nerve Sheath Tumors. Oncologist (2019) 24(6):857-63. doi: 10.1634/theoncologist.2018-0160

13. Kim S, Kim MY, Koh J, Go H, Lee DS, Jeon YK, et al. Programmed Death-1 Ligand 1 and 2 are Highly Expressed in Pleomorphic Carcinomas of the Lung: Comparison of Sarcomatous and Carcinomatous Areas. Eur J Cancer (2015) 51(17):2698-707. doi: 10.1016/j.ejca.2015.08.013

14. Xia L, Liu Y, Wang Y. PD-1/PD-L1 Blockade Therapy in Advanced nonSmall-Cell Lung Cancer: Current Status and Future Directions. Oncologist (2019) 24(Suppl 1):S31-41. doi: 10.1634/theoncologist.2019-IO-S1-s05

15. Huang J, Xu B, Mo H, Zhang W, Chen X, Wu D, et al. Safety, Activity, and Biomarkers of SHR-1210, an Anti-PD-1 Antibody, for Patients With Advanced Esophageal Carcinoma. Clin Cancer Res (2018) 24(6):1296-304. doi: 10.1158/1078-0432.CCR-17-2439

16. Xu J, Zhang Y, Jia R, Yue C, Chang L, Liu R, et al. Anti-PD-1 Antibody SHR-1210 Combined With Apatinib for Advanced Hepatocellular Carcinoma, Gastric, or Esophagogastric Junction Cancer: An Open-label, Dose Escalation and Expansion Study. Clin Cancer Res (2019) 25(2):515-23. doi: 10.1158/1078-0432.CCR-18-2484

17. Velcheti V, Rimm DL, Schalper KA. Sarcomatoid Lung Carcinomas Show High Levels of Programmed Death Ligand-1 (PD-L1). J Thorac Oncol (2013) 8 (6):803-5. doi: 10.1097/JTO.0b013e318292be18

18. Sun Y, Niu W, Du F, Du C, Li S, Wang J, et al. Safety, Pharmacokinetics, and Antitumor Properties of Anlotinib, an Oral Multi-Target Tyrosine Kinase Inhibitor, in Patients With Advanced Refractory Solid Tumors. J Hematol Oncol (2016) 9(1):105. doi: 10.1186/s13045-016-0332-8

Conflict of Interest: The authors declare that the research was conducted in the absence of any commercial or financial relationships that could be construed as a potential conflict of interest.

Copyright (C) $2021 \mathrm{Li}$, Liang, He, Sui and Qin. This is an open-access article distributed under the terms of the Creative Commons Attribution License (CC BY). The use, distribution or reproduction in other forums is permitted, provided the original author(s) and the copyright owner(s) are credited and that the original publication in this journal is cited, in accordance with accepted academic practice. No use, distribution or reproduction is permitted which does not comply with these terms. 